\title{
Downregulation of matrix metalloproteinase-9 mRNA by valproic acid plays a role in inhibiting the shedding of MHC class I-related molecules A and B on the surface of human osteosarcoma cells
}

\author{
KOJI YAMANEGI $^{1 *}$, JUNKO YAMANE ${ }^{1 *}$, KENTA KOBAYASHI $^{1}$, HIDEKI OHYAMA ${ }^{1}$, \\ KEIJI NAKASHO ${ }^{1}$, NAOKO YAMADA ${ }^{1}$, MASAKI HATA ${ }^{1}$, SATORU FUKUNAGA ${ }^{2}$, \\ HIROYUKI FUTANI ${ }^{2}$, HARUKI OKAMURA ${ }^{3}$ and NOBUYUKI TERADA ${ }^{1}$ \\ Departments of ${ }^{1}$ Pathology, ${ }^{2}$ Orthopedic Surgery and ${ }^{3}$ Tumor Immunology and Cell Therapy, \\ Hyogo College of Medicine, Nishinomiya, Hyogo 663-8501, Japan
}

Received March 7, 2012; Accepted June 15, 2012

DOI: 10.3892/or.2012.1981

\begin{abstract}
Valproic acid, a histone deacetylase inhibitor, increases the expression of cell surface MHC class I-related chain molecules (MICs) A and B (MICA and B) in osteosarcoma cells and decreases their secretion of soluble MICA and MICB, which are produced by the proteolytic cleavage of cell surface MICs. Osteosarcoma cells have been reported to produce high levels of matrix metalloproteinase (MMP)-2 and -9. In this study, we investigated the involvement of MMP-2 and -9 in the inhibitory action of valproic acid (VPA) on the proteolytic cleavage of cell surface MICs using the U-2 OS and SaOS-2 osteosarcoma cell lines. VPA caused a marked decrease in the expression of MMP-9 mRNA in the U-2 OS and SaOS- 2 cells and in the expression of MMP-2 mRNA in the U-2 OS cells, but only a slight decrease in the expression of MMP-2 mRNA in the SaOS-2 cells. The transfection of small interfering RNA (siRNA) for MMP-9 decreased the secretion of soluble MICA and MICB by both U-2 OS and SaOS-2 cells, but that of siRNA for MMP-2 did not. The present study therefore demonstrates that the downregulation of MMP-9 mRNA by VPA plays a role in the inhibitory action of VPA on the secretion of soluble MICA and MICB in osteosarcoma cells.
\end{abstract}

\section{Introduction}

Tumor cells express MHC class I-related chain molecules (MICs) A and B (MICA and MICB), which are ligands of the

Correspondence to: Dr Koji Yamanegi, Department of Pathology, Hyogo College of Medicine, 1-1 Mukogawa-cho, Nishinomiya, Hyogo 663-8501, Japan

E-mail: yamanegi@hyo-med.ac.jp

*Contributed equally

Key words: valproic acid, histone deacetylase inhibitor, MHC class I-related chain molecule, metalloproteinase, osteosarcoma
NKG2D receptor that is expressed on the surface of cytotoxic immune cells, such as natural killer (NK), $\gamma \delta^{+} \mathrm{T}$ and $\mathrm{CD}^{+} \alpha \beta^{+}$ $\mathrm{T}$ cells $(1,2)$. The binding of the NKG2D receptor to its ligands activates $\mathrm{NK}$ and $\gamma \delta^{+} \mathrm{T}$ cells, and co-stimulates tumor-antigenspecific $\mathrm{CD}^{+} \alpha \beta^{+} \mathrm{T}$ cells $(1,2)$. Therefore, the NKG2D-MIC system plays an important role in the cytotoxicity of immune cells. However, tumor cells produce soluble MICs and thus are able to avoid being attacked by cytotoxic immune cells. Soluble MICs, which are produced by the proteolytic cleavage of their extracellular domain by proteases $(1,3-6)$, interfere with the binding of MICs on the surface of tumor cells to NKG2D receptors on the surface of cytotoxic immune cells, and the binding of soluble MICs to NKG2D receptors downregulates the NKG2D receptors on the surface of cytotoxic immune cells $(1,6-9)$.

The gene expression in tumor cells is altered by both genetic and epigenetic events, and epigenetic modifiers, such as histone deacetylase (HDAC) and DNA methylation inhibitors, alter their gene expression profiles $(10,11)$. A number of studies have demonstrated that HDAC inhibitors stimulate the expression of cell surface MICA and MICB in a variety of tumors (12-15). Valproic acid (VPA), a HDAC inhibitor, increases the expression of MICA and MICB on the surface of human osteosarcoma cells (16). Furthermore, VPA decreases the production of soluble MICA and MICB in these cells (16). However, the mechanisms by which VPA decreases the production of soluble MICA and MICB in osteosarcoma cells remains to be elucidated.

Soluble MICA and MICB are produced by the proteolytic cleavage of cell surface MICA and MICB as broad-spectrum metalloproteinase inhibitors suppress the shedding of MICs (3-6). Human osteosarcoma cells produce high levels of matrix metalloproteinase (MMP)-2 and -9 (17,18), and HDAC inhibitors, including VPA have been shown to decrease the expression of MMP-2 and -9 in thyroid, gastric and lung cancer cells (19-21). Therefore, in this study, we investigated the effect of VPA on the mRNA expression of these MMPs in osteosarcoma cells and the roles of these MMPs in the cleavage of MICA and MICB on the cell surface. The present study shows that the downregulation of MMP-9 mRNA by VPA is involved 
in the inhibitory action of VPA on the shedding of MICA and MICB on the surface of human osteosarcoma cells.

\section{Materials and methods}

Reagents and antibodies. Sodium valproate was purchased from Wako (Osaka, Japan), the PE-conjugated anti-human MICA/B mouse monoclonal antibody $\left(\operatorname{IgG}_{2 b}\right)$ from $R \& D$ Systems (Minneapolis, MN, USA) and the control mouse $\mathrm{IgG}_{2 \mathrm{~b}}$ from BioLegend (San Diego, CA, USA). The MMP-2/MMP-9 inhibitor (an inhibitor of MMP-2 and -9), GM 6001 (a broadspectrum inhibitor of MMPs), and the GM 6001 negative control were purchased from Calbiochem (Merck, Tokyo, Japan).

Cells. U-2 OS and SaOS-2 human osteosarcoma cells were purchased from the American Type Culture Collection (ATCC, Manasas, VA, USA), and Riken BRC Cell Bank (Tsukuba, Ibaragi, Japan), respectively. The U-2 OS and SaOS-2 cells were cultured in McCoy's 5A modified medium (Invitrogen, Carlsbad, CA, USA). All these media contained $10 \%$ fetal bovine serum (FBS) (MP Biomedical, Inc., Morgan Irvine, CA, USA), penicillin (100 U/ml) and streptomycin $(100 \mu \mathrm{g} / \mathrm{ml})$. All cells were cultured in a humidified atmosphere of $5 \% \mathrm{CO}_{2}$ in air at $37^{\circ} \mathrm{C}$.

Flow cytometric analysis. U-2 OS and SaOS-2 cells were seeded at 2 and $4 \times 10^{3}$ cells/dish, respectively, in $6 \mathrm{~cm}$-tissue culture dishes containing $3 \mathrm{ml}$ of medium/dish. After $24 \mathrm{~h}$ (day 0), VPA was added to the medium at $1.0 \mathrm{mM}$, and the cells were cultured for another 7 days, with a medium change on day 3. The cells were detached from the dishes, and the expression of membrane-bound MICA/B was analyzed by a flow cytometric analysis, as described previously (16). The percentages of membrane-bound MICA/B-positive cells were determined by flow cytometric analysis, and the effects of VPA were evaluated by determining the ratio of the percentage of positive cells in the treated cultures to the average percentage of positive cells in the untreated control cultures.

Enzyme-linked immunosorbent assays (ELISAs). U-2 OS and SaOS-2 cells were seeded at 1 and $2 \times 10^{5}$ cells/dish, respectively, in $10 \mathrm{~cm}$-tissue culture dishes containing $5 \mathrm{ml}$ of medium/dish. After $24 \mathrm{~h}$ (day 0), VPA was added to the medium at $1.0 \mathrm{mM}$, and the cells were cultured for another 7 days, with a medium change on day 3 . The medium was collected for an assay of the soluble MICA and soluble MICB levels using ELISA systems for human soluble MICA and MICB (R\&D Systems) and a microplate reader (Bio-Rad Laboratories, Tokyo, Japan). The amount of soluble MICA or MICB $/ 10^{4}$ viable cells in the treated cultures was expressed as a ratio of the average value in the untreated control cultures.

Treatment with MMP inhibitors. U-2 OS and SaOS-2 cells were seeded at 2 and $4 \times 10^{5}$ cells/dish, respectively, in $10 \mathrm{~cm}$-tissue culture dishes containing $5 \mathrm{ml}$ of medium/dish. After $24 \mathrm{~h}$, VPA (1.0 mM), MMP-2/MMP-9 inhibitor (10 $\mu \mathrm{M})$, GM6001 (a broad-spectrum inhibitor of MMPs) $(2 \mu \mathrm{M})$ or the GM 6001 negative control $(2 \mu \mathrm{M})$ was added to the medium and the cells were cultured for another $48 \mathrm{~h}$. The soluble MICA and MICB levels in the medium were assayed using the ELISA systems as described above. The amount of soluble MICA or MICB $/ 10^{4}$ viable cells in the treated cultures was expressed as a ratio of the average value in the untreated control cultures.

Quantitative real-time PCR. U-2 OS and SaOS-2 cells were cultured in medium with or without $1.0 \mathrm{mM}$ VPA, for 7 days, with a medium change on day 3 , and on days 3 and 7 , the total RNA was extracted from the cells in each culture dish with TRIzol reagent (Invitrogen). An aliquot of RNA was reverse-transcribed using Superscript II reverse transcriptase (Invitrogen) according to the manufacturer's instructions. Real-time PCR for MMP-2, -9 or -14, or a disintegrin and metalloproteinase (ADAM)-17 mRNA was performed using TaqMan Gene Expression assays (Applied Biosystems, Foster City, CA, USA). The primer sets used were Hs01548733_ml for MMP-2 mRNA, Hs00957562_ml for MMP-9 mRNA, Hs00237119_ml for MMP-14 mRNA and Hs01041915_ml for ADAM-17 mRNA (Applied Biosystems). The amount of GAPDH mRNA as an internal reference was estimated using human GAPDH as the endogenous control (Applied Biosystems), and the amount of MMP-2, -9 or -14 , or ADAM-17 mRNA in each sample was corrected by the amount of GAPDH mRNA in the corresponding sample. The amount of MMP-2, -9 or -14 , or ADAM-17 mRNA in the treated cultures was expressed as a ratio of the average value in the untreated control cultures.

Effects of small interfering RNA (siRNA) for MMP-2 or -9 on the secretion of soluble MICA and MICB. The siRNAs designed for MMP-2 and -9 mRNAs were 5'-GGAAAGAUUGAUGC GGUAtt-3' (sense strand) and 5'-CAUCACCUAUUGGAUCC AAtt-3' (sense strand), respectively, and were synthesized by Applied Biosystems. U-2 OS and SaOS-2 cells were seeded at 1 and $2 \times 10^{5}$ cells/well, respectively, in 6 well-tissue culture plates and cultured in $2 \mathrm{ml}$ of medium for $24 \mathrm{~h}$. The culture medium was changed to Opti-MEM medium (Invitrogen), and the cells were transfected with $10 \mathrm{nM}$ of MMP-2 or MMP-9 siRNA and negative control siRNA using an RNAiMAX reagent (Invitrogen), and were cultured for another $48 \mathrm{~h}$. The medium was collected, and the levels of soluble MICA and MICB in the medium were determined as described above, and the expression of MMP-2 and -9 mRNA in the cells was examined by reverse transcription polymerase chain reaction (RT-PCR). The amount of soluble MICA or $\mathrm{MICB} / 10^{4}$ viable cells in the treated cultures was expressed as a ratio of the average value in the untreated control cultures.

$R T-P C R$. Total cell RNA was extracted from the U-2 OS and SaOS-2 cells using TRIzol reagent (Invitrogen) according to the manufacturer's instructions. The reverse transcription of $2 \mu \mathrm{g}$ of total RNA was performed at $42^{\circ} \mathrm{C}$ for $1 \mathrm{~h}$ using random primers (Roche Applied Science, Indianapolis, IN, USA) and Transcriptor Reverse Transcriptase (Roche Applied Science), and cDNAs produced were sequentially amplified by PCR with Takara Ex Taq ${ }^{\mathrm{TM}}$ DNA polymerase (Takara Bio, Inc., Ohtsu, Shiga, Japan) using specific primer sets as follows: sense, 5'-ACGATGATGACCGCAAGTGG-3' and antisense, 5'-GGA GCTCAGGCCAGAATGTG-3' for MMP-2; sense, 5'-AGGAC GGCAATGCTGATGGG-3' and antisense, 



Figure 1. Effects of VPA on the expression of cell surface MICA/B and the secretion of soluble MICA and MICB. U-2 OS and SaOS-2 cells were cultured in medium with or without $1.0 \mathrm{mM}$ VPA for 7 days, with a medium change on day 3. (A) The expression of cell surface MICA/B was analyzed by flow cytometry using an antibody that recognizes both MICA and MICB, and is shown as the ratio of a percentage of positive cells in the treated cultures to the average percentage of positive cells in the control cultures. Each bar indicates the mean + SE of 6 dishes. (B and C) Effects of VPA on the secretion of (B) soluble MICA and (C) soluble MICB. The amount of soluble MICA and MICB accumulated in the medium during the last 4 days of a 7-day culture (from days 3 to 7 ) was assayed and the amount of soluble MICA or MICB $/ 10^{4}$ viable cells was determined. The values are expressed as a ratio to the average amount of soluble MICA or MICB $/ 10^{4}$ viable cells in control cultures. Each bar indicates the mean $\pm \mathrm{SE}$ of 8 dishes. ${ }^{*} \mathrm{P}<0.05$, significant difference from the values of the control.

5'-GAGGTGCCG GATGCCATTCA-3' for MMP-9; and sense, 5'-GTCATCAAT GGAAATCCCATCACC-3' and antisense, 5'-GCTCAGGGAT GACCTTGCCC-3' for GAPDH. The amplification conditions of the PCR for MMP-2 and -9 were 25 cycles at $95^{\circ} \mathrm{C}$ for $30 \mathrm{sec}, 55^{\circ} \mathrm{C}$ for $30 \mathrm{sec}$, and $72^{\circ} \mathrm{C}$ for $1 \mathrm{~min}$, followed by heating at $72^{\circ} \mathrm{C}$ for $7 \mathrm{~min}$, and that for GAPDH was 25 cycles at $95^{\circ} \mathrm{C}$ for $30 \mathrm{sec}, 57^{\circ} \mathrm{C}$ for $30 \mathrm{sec}$, and $72^{\circ} \mathrm{C}$ for $1 \mathrm{~min}$, followed by heating at $72^{\circ} \mathrm{C}$ for $7 \mathrm{~min}$. The amplified fragments were resolved by electrophoresis on $1.5 \%$ agarose gels, and were detected by ethidium bromide staining.

Statistical analysis. The data are presented as the means + SE. The data of 2 groups were analyzed by the Student's t-test, and the data of 3 groups or more by the two-tailed Dunnett's t-test for multiple comparisons. A P-value $<0.05$ was considered to indicate a statistically significant difference.

\section{Results}

Expression of $M I C A / B$ on the surface of osteosarcoma cells and secretion of soluble MICA and MICB. The osteosarcoma cells were cultured in the presence or absence of VPA $(1.0 \mathrm{mM})$ for 7 days, and the cell surface expression of MICA/B was examined by flow cytometry (Fig. 1A). Cell surface MICA/B was expressed in 7.2 and $5.1 \%$ of the U-2 OS and SaOS-2 cells, respectively. VPA increased the expression of MICA/B on the surface of U-2 OS and SaOS-2 cells by approximately 5.0- and 2.6-fold, respectively.

The amount of soluble MICA or MICB in the medium of the osteosarcoma cells during the last 4 days of a 7-days culture (from days 3 to 7) was estimated (Fig. 1B and C). VPA (1.0 mM) significantly decreased the amount of both soluble MICA and MICB in the culture medium of the 2 osteosarcoma cell lines.

Role of MMP in the secretion of soluble MICA and MICB. Soluble MICA and MICB are produced by the proteolytic cleavage of cell surface MICA and MICB and human osteosarcoma cells produce MMP-2 and -9. Therefore, the effects of GM6001 (a broad-spectrum metalloproteinase inhibitor) and MMP-2/MMP-9 inhibitor (an inhibitor of MMP-2 and -9) on the shedding of MICA and MICB were examined (Fig. 2). The MMP-2/MMP-9 inhibitor $(10 \mu \mathrm{M})$ and GM $6001(2 \mu \mathrm{M})$ as well as VPA (1 mM) decreased the amount of soluble MICA and MICB in the U-2 OS and SaOS-2 cells.

Subsequently, the effects of VPA on the expression of MMP-2, -9 and -14, and ADAM-17 mRNA in U-2 OS and SaOS- 2 cells cultured for 3 or 7 days were examined by quantitative real-time PCR. VPA $(1.0 \mathrm{mM})$ markedly decreased the expression of MMP-9 mRNA in the U-2 OS and SaOS-2 cells (Fig. 3). VPA also markedly decreased the expression of 


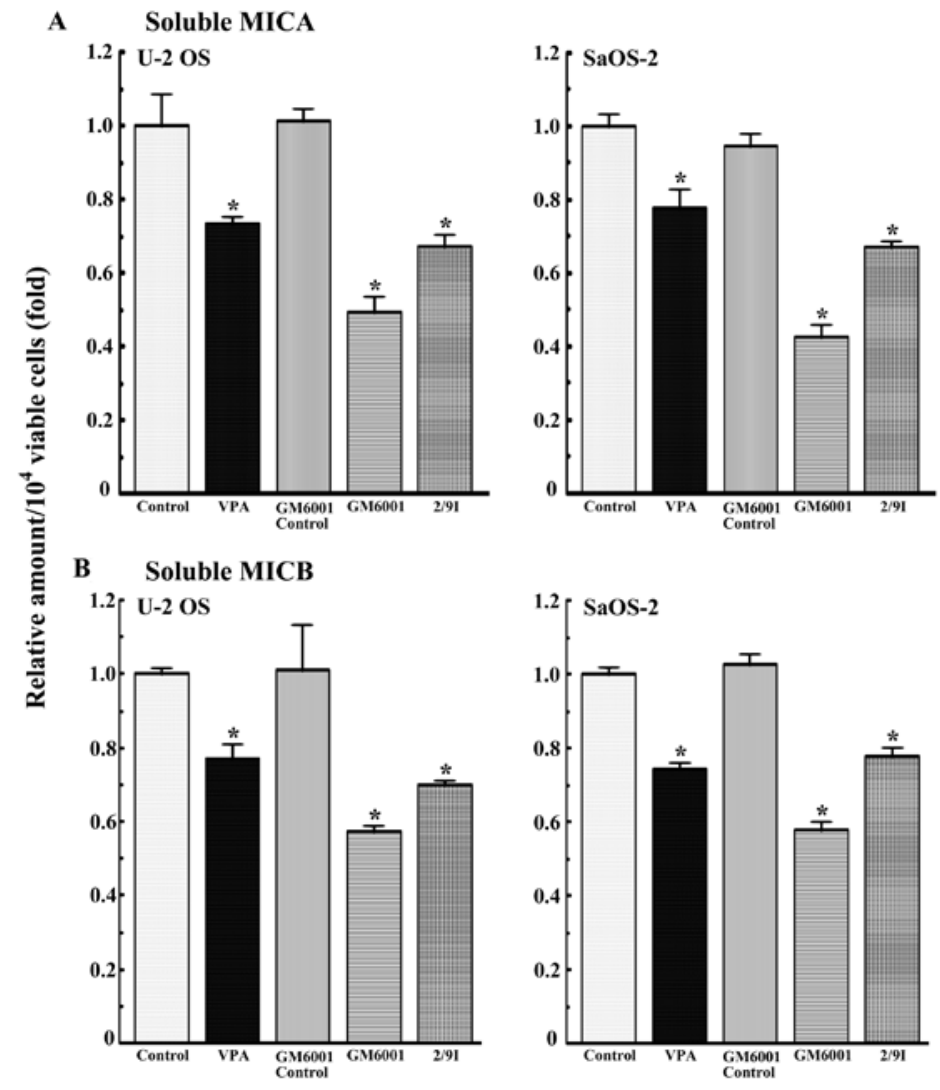

Figure 2. Effects of matrix metalloproteinase (MMP) inhibitors on the secretion of soluble MICA and MICB. U-2 OS and SaOS-2 cells were cultured in medium with VPA $(1.0 \mathrm{mM})$, the inhibitor of MMP-2 and -9 (2/9I) (10 $\mu \mathrm{M})$, GM6001 (a broad-spectrum inhibitor of MMPs) $(2 \mu \mathrm{M})$ and the GM6001 negative control (GM6001 control) $(2 \mu \mathrm{M})$ for $48 \mathrm{~h}$. The amount of soluble MICA or MICB accumulated in the medium was assayed and the amount of soluble MICA or $\mathrm{MICB} / 10^{4}$ viable cells was determined. The values are expressed as a ratio to the average amount of soluble MICA or MICB $/ 10^{4}$ viable cells in the control cultures. Each bar indicates the mean \pm SE of 6 dishes. ${ }^{*} \mathrm{P}<0.05$, significant difference from the values of the control.
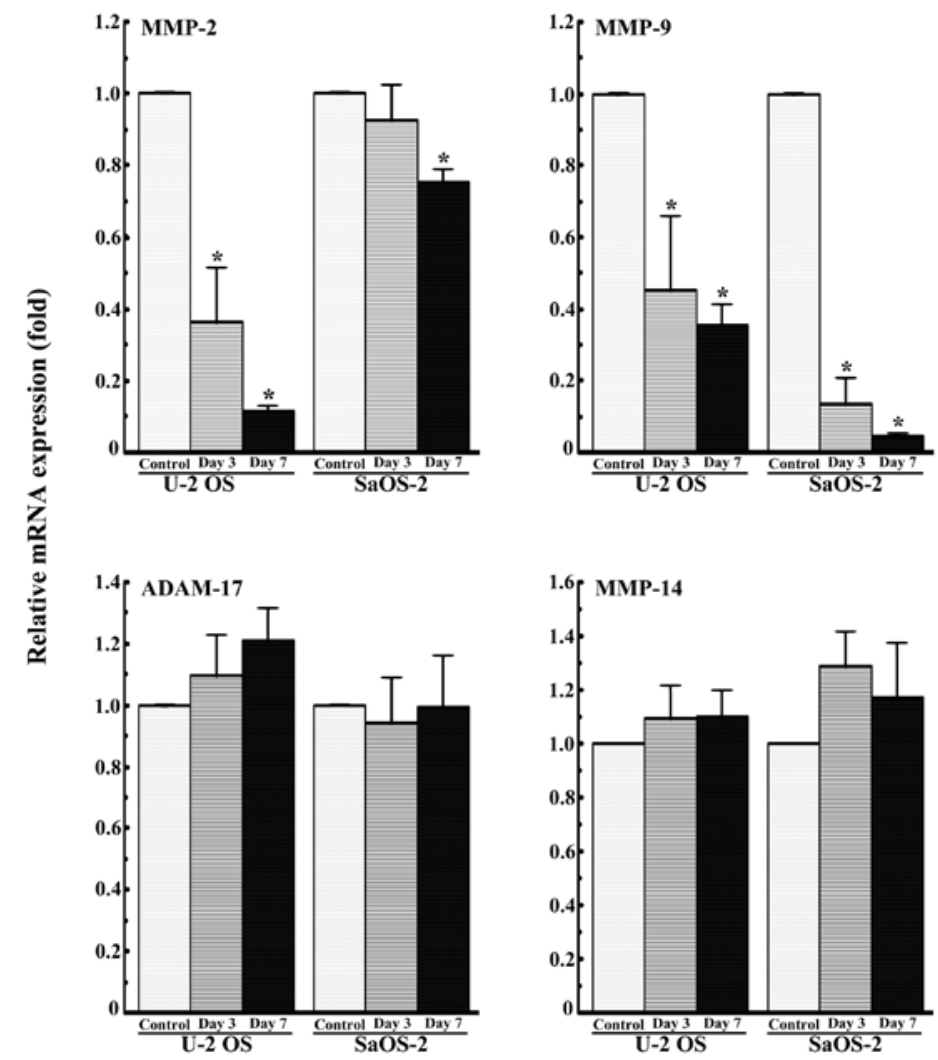

Figure 3. Effects of VPA on the expression of MMP-2, -9 and -14 and ADAM-17 mRNA. U-2 OS and SaOS-2 cells were cultured in medium with or without $1.0 \mathrm{mM}$ VPA for 3 or 7 days, and the expression of MMP-2, -9 and -14 and ADAM-17 mRNA was examined by real-time PCR. The values are expressed as the ratio to the average value in control cultures. The bar indicates the mean $\pm \mathrm{SE}$ of 8 dishes. $\mathrm{P}<0.05$, significant difference from the values of the control. 
A

\begin{tabular}{|c|c|c|c|c|c|c|c|c|}
\hline \multicolumn{4}{|c|}{$\mathrm{U}-2$ OS cells } & & \multicolumn{4}{|c|}{ SaOS-2 cells } \\
\hline & & MI & & & MI & & & P-9 \\
\hline cont & siRNA & cont & SiRNA & & cont & siRNA & cont & SiRNA \\
\hline$=$ & & $=$ & & & $=$ & 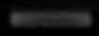 & es & . \\
\hline 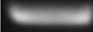 & 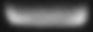 & 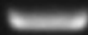 & 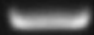 & GAPDH & 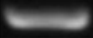 & 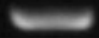 & 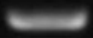 & E \\
\hline
\end{tabular}

B

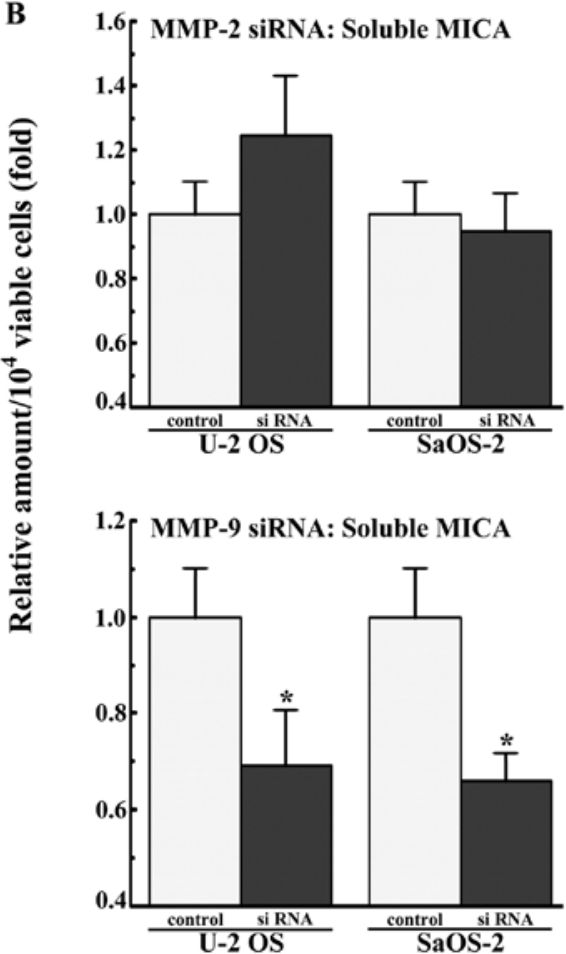

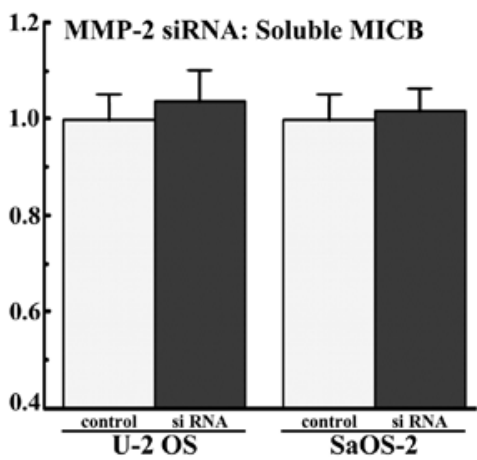

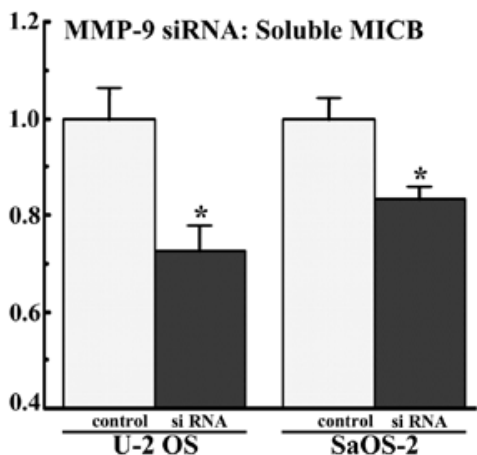

Figure 4. Role of MMP-9 or MMP-2 in the production of soluble MICA and MICB. (A) Effects of the siRNA for MMP-2 or -9 on the mRNA expression levels of MMP-2 or -9. The expression of MMP-2 and -9 mRNA in the U-2 OS and SaOS-2 cells was examined by RT-PCR after the transfection with siRNA for $48 \mathrm{~h}$. (B) Effects of siRNA for MMP-2 or -9 on the secretion of soluble MICA and MICB. U-2 OS and SaOS-2 cells were transfected with siRNA for MMP-2 or -9, and were cultured for $48 \mathrm{~h}$. The amount of soluble MICA or MICB accumulated in the medium was assayed and the amount of soluble MICA or $\mathrm{MICB} / 10^{4}$ viable cells was determined. The average amount of soluble MIC or MICB $/ 10^{4}$ viable cells in control cultures is expressed as 1.0 . The bar indicates the mean \pm SE of 8 wells. ${ }^{*} \mathrm{P}<0.05$, significant difference from the values of the control.

MMP-2 mRNA in the U-2 OS cells, but showed little effect on the expression of MMP-2 mRNA in the SaOS-2 cells (Fig. 3). VPA did not decrease the expression of MMP-14 and ADAM-17 mRNA (Fig. 3).

The U-2 OS and SaOS-2 cells were transfected with siRNAs for MMP-2 and -9, and the amount of soluble MICA and MICB in the medium was assayed after 2 days of culture. siRNAs for MMP-2 and -9 markedly decreased the expression levels of these mRNAs (Fig. 4A). siRNA for MMP-9 decreased the amounts of soluble MICA and MICB secreted by the U-2 OS and SaOS-2 cells by approximately $20-30 \%$, while siRNA for MMP-2 did not (Fig. 4B).

\section{Discussion}

Culture with 1.0 mM VPA increased the expression of MICA/B on the surface of osteosarcoma cells and inhibited their secretion of soluble MICA and MICB, confirming the results of our previous report (16). Our previous study showed that $1.0 \mathrm{mM}$ VPA increased the acetylation of histones, suggesting that at least a part of the action of VPA can be ascribed to its action as a HDAC inhibitor (16).
GM6001 (a broad-spectrum inhibitor of MMPs) and the inhibitor of MMP-2 and -9 decreased the secretion of MICA and MICB by osteosarcoma cells and siRNA for MMP-9 decreased the secretion of MICA and MICB, whereas that for MMP-2 did not. These results indicate that MMP-9 is responsible for the shedding of MICA and MICB on the surface of osteosarcoma cells. Several proteases have been reported to be responsible for the shedding of cell surface MICA and MICB; MMP-14 for MICA, ADAM-10 for MICA and ADAM-17 for MICA and MICB $(6,22,23)$. Therefore, these proteases may also be responsible for the shedding of MICA and MICB on the surface of osteosarcoma cells.

VPA markedly decreased the expression of MMP-9 mRNA in the osteosarcoma cells, consistent with previous studies using other types of cancer cells (19-21). Therefore, the inhibitory action of VPA on the secretion of soluble MICA and MICB is ascribed at least in part to the downregulation of MMP-9 mRNA by VPA. VPA did not affect the expression of MMP-14 and ADAM-17 mRNA. However, other proteases, including ADAM-10 may be related to the inhibitory action of VPA on the shedding of MICA and MICB on the surface of osteosarcoma cells. 
In conclusion, the present study shows that the downregulation of MMP-9 mRNA by VPA is involved in the inhibitory action of VPA on the secretion of soluble MICA and MICB from the surface of osteosarcoma cells. To our knowledge, this is the first study to demonstrate that MMP-9 plays a role in the shedding of MICA and MICB from the surface of tumor cells.

\section{Acknowledgements}

This study was supported in part by a Grant-in-Aid for Young Scientists (B) (23792159) from the Ministry of Education, Culture, Sports, Science and Technology of Japan, a Strategic Program Grant for Research Infrastructure Development in Private Institutes and a Grant-in-Aid for Promotion of Technical Seeds in Advanced Medicine, the Hyogo College of Medicine.

\section{References}

1. Waldhauer I and Steinle A: NK cells and cancer immunosurveillance. Oncogene 27: 5932-5943, 2008.

2. Nausch $\mathrm{N}$ and Cerwenka A: NKG2D ligands in tumor immunity. Oncogene 27: 5944-5958, 2008.

3. Salih HR, Rammensee HG and Steinle A: Cutting edge: downregulation of MICA on human tumors by proteolytic shedding. J Immunol 169: 4098-4102, 2002.

4. Salih HR, Goehlsdorf D and Steinle A: Release of MICB molecules by tumor cells: mechanism and soluble MICB in sera of cancer patients. Hum Immunol 67: 188-195, 2006.

5. Waldhauer I and Steinle A: Proteolytic release of soluble UL16binding protein 2 from tumor cells. Cancer Res 66: 2520-2526, 2006.

6. Boutet P, Agüera-González S, Atkinson S, Pennington CJ, Edwards DR, Murphy G, Reyburn HT and Valés-Gómez M: Cutting edge: the metalloproteinase ADAM17/TNF- $\alpha$-converting enzyme regulates proteolytic shedding of the MHC class I-related chain B protein. J Immunol 182: 49-53, 2009.

7. Groh V, Wu J, Yee C and Spies T: Tumour-derived soluble MIC ligands impair expression of NKG2D and T-cell activation. Nature 419: 734-738, 2002.

8. Raffaghello L, Prigione I, Airoldi I, Camoriano M, Levreri I, Gambini C, Pende D, Steinle A, Ferrone S and Pistoia V: Downregulation and/or release of NKG2D ligands as immune evasion strategy of human neuroblastoma. Neoplasia 6: 558-568, 2004.

9. Märten A, von Lilienfeld-Toal M, Büchler MW and Schmidt J: Soluble MIC is elevated in the serum of patients with pancreatic carcinoma diminishing $\gamma \delta \mathrm{T}$ cell cytotoxicity. Int J Cancer 119: 2359-2365, 2006.

10. Yoo CB and Jones PA: Epigenetic therapy of cancer: past, present and future. Nat Rev Drug Discov 5: 37-50, 2006.

11. Kristensen LS, Nielsen HM and Hansen LL: Epigenetics and cancer treatment. Eur J Pharmacol 625: 131-142, 2009.
12. Armeanu S, Bitzer M, Lauer UM, Venturelli S, Pathil A, Krusch M, Kaiser S, Jobst J, Smirnow I, Wagner A, et al: Natural killer cell-mediated lysis of hepatoma cells via specific induction of NKG2D ligands by the histone deacetylase inhibitor sodium valproate. Cancer Res 65: 6321-6329, 2005.

13. Schmudde M, Braun A, Pende D, Sonnenmann J, Klier U, Beck JF, Moretta L and Bröker BM: Histone deacetylase inhibitors sensitize tumour cells for cytotoxic effects of natural killer cells. Cancer Lett 272: 110-121, 2008.

14. Zhang C, Wang Y, Zhou Z, Zhang J and Tian Z: Sodium butyrate upregulates expression of NKG2D ligand MICA/B in HeLa and HepG2 cell lines and increases their susceptibility to NK lysis. Cancer Immunol Immunother 58: 1275-1285, 2009.

15. Poggi A, Catellani S, Garuti A, Pierri I, Gobbi M and Zocchi MR: Effective in vivo induction of NKG2D ligands in acute myeloid leukaemias by all-trans-retinoic acid or sodium valproate. Leukemia 23: 641-648, 2009.

16. Yamanegi K, Yamane J, Kobayashi K, Kato-Kogoe N, Ohyama H, Nakasho K, Yamada N, Hata M, Nishioka T, Fukunaga S, et al: Sodium valproate, a histone deacetylase inhibitor, augments the expression of cell-surface NKG2D ligands, MICA/B, without increasing their soluble forms to enhance susceptibility of human osteosarcoma cells to NK cell-mediated cytotoxicity. Oncol Rep 24: 1621-1627, 2010.

17. Cho HJ, Lee TS, Park JB, Park KK, Choe JY, Sin DI, Park YY, Moon YS, Lee KG, Yeo JH, et al: Disulfiram suppresses invasive ability of osteosarcoma cells via the inhibition of MMP-2 and MMP-9 expression. J Biochem Mol Biol 40: 1069-1076, 2007.

18. Xin ZF, Kim YK and Jung ST: Risedronate inhibits human osteosarcoma cell invasion. J Exp Clin Cancer Res 28: 105, 2009.

19. Lee KH, Choi EY, Kim MK, Kim KO, Jang BI, Kim SW, Kim SW, Song SK and Kim JR: Inhibition of histone deacetylase activity down-regulates urokinase plasminogen activator and matrix metalloproteinase-9 expression in gastric cancer. Mol Cell Biochem 343: 163-171, 2010.

20. Mitmarker EJ, Griff NJ, Grogan RH, Sarkar R, Kebebew E, Duh QY, Clark OH and Shen WT: Modulation of matrix metalloproteinase activity in human thyroid cancer cell lines using demethylating agents and histone deacetylase inhibitors. Surgery 149: 504-511, 2011.

21. Vinodhkumar R, Song YS, Ravikumar V, Ramakrishnan G and Devaki T: Depsipeptide a histone deacetylase inhibitor down regulates levels of matrix metalloproteinases 2 and $9 \mathrm{mRNA}$ and protein expressions in lung cancer cells (A549). Chem Biol Interact 165: 220-229, 2007.

22. Waldhauer I, Goehlsdorf D, Gieseke F, Weinschenk T, Wittenbrink M, Ludwig A, Stevanovic S, Rammensee HG and Steinle A: Tumor-associated MICA is shed by ADAM proteases. Cancer Res 68: 6368-6376, 2008.

23. Liu G, Atteridge CL, Wang X, Lundgren AD and Wu JD: The membrane type matrix metalloproteinase MMP14 mediates constitutive shedding of MHC class I chain-related molecule A independent of a disintegrin and metalloproteinases. J Immunol 184: 3346-3350, 2010. 